bination of the ten states obtained by permuting the three colour labels among three otherwise identical strange quarks.

The rule yields just the colour combinations that can naturally occur, no more and no fewer. But there are also more direct tests of the idea. Among other things, the magnetic moment of the neutron is a direct consequence of the spin of the $u$ quark, given that the Pauli principle requires that the spins of the two accompanying $d$ quarks must be oppositely aligned. Moreover, there are many particle collisions in which the likelihood of a particular outcome is a function of the number of colour states of the underlying quarks, so that the scale of the quantum number can be measured directly.

Successful though quantum chromodynamics (QCD) may be in classifying hadrons, it is less successful as a means of accurate computation than the electroweak theory, and for two inescapable reasons. First, the interaction it deals with is the 'strong' interaction, which leads directly to the consequence that the first approximation to the solution of equations (by perturbation methods) may not be not a good approximation. (The electroweak theory has the benefit that the successive terms in approximate calculations are proportional to successive powers of the dimensionless fine-structure constant $2 \pi e^{2} / h c$, numerically approximately $1 / 137$.) Second, because the particles that mediate the force, the gluons, carry colour charge, they interact with each other, giving the theory an awkward nonlinearity.

The computational difficulties have led to a way of calculating QCD processes by means of lattice models, in which the fields of quarks and gluons are represented by their (variable) values at the vertices of a manageably small lattice. (The technique was developed by K. G. Wilson, who was awarded a Nobel prize for his work in 1982.) Perhaps the remarkable feature of this technique is that it yields good approximations so often.

Whether the theory is philosophically compelling is another matter. As with the electroweak theory, which would allow the existence of the unphysical righthanded neutrino, QCD could accommodate a greater range of particles than are known to exist. The origin of the restrictions found in nature is empirical, not a natural feature of the theory. Moreover, the theory will not be tested properly until the top quark is definitively found; hints of the discovery earlier this year from Fermilab have yet to be confirmed. The latest estimates, inferred from the production of more than a million $\mathrm{Z}^{0}$ and $\mathrm{W}^{ \pm}$ particles at CERN, suggest that the mass of the top quark is within 10 per cent of $172 \mathrm{GeV}$.

\title{
Vital yardstick hidden still
}

THE Higgs boson, not yet discovered, is arguably the most important of all particles in contemporary particle physics. To the extent that it is an indispensable part of the electroweak theory of the electromagnetic and the weak nuclear forces, the theory will not be properly tested until the particle has been found. That is especially important because the Higgs particle is a strictly theoretical construct.

One practical difficulty is that there is no unambiguous estimate of the mass of the Higgs particle, although the failure to find it so far suggests that the mass must exceed that of, say, the $\mathrm{W}^{-}$. now copiously produced at particle accelerators of sufficient energy.

The role of the Higgs particle is abstract: to create the asymmetry between left- and right-handed particles that is the hallmark of the electroweak theory. How can the mere existence of a particle (or, more strictly, of the field of which the particle is a manifestation) accomplish that? It can, if the state in which the field is everywhere zero is not the state of lowest energy.

That sounds wrong, but is not impossible. Particle physics is ready with homely examples. Thus a ferromagnetic material below its Curie temperature will usually consist of domains in which atomic nuclei are ferromagnetically ordered even when the overall magnetization is zero. In the process, both the translational and rotational symmetry of the solid are lost. The symmetry is said to be "spontaneously broken".

In the electroweak interaction, the nonzero Higgs field is the analogue of the domain-structured texture of a ferromagnetic material below its Curie temperature. The asymmetry is the origin of the left-handedness of the neutrino. But the Higgs field also interacts both with the massless photon and with the intermediate vector bosons, $W^{+}$and $Z^{0}$. One effect of that interaction is to give these mediating particles their characteristic (and very different) masses.

Plausible though it may be, the concept of the Higgs field has startling implications for what is meant by the physical vacuum, and in particular for the notion that the vacuum is literally empty. In the immediate neighbourhood of a lepton, it appears that there will be a non-zero Higgs field whose presence determines the magnitude of its interactions with other particles.

The idea that the physical vacuum may be the source of physical particles is not all that novel, of course. A sufficiently energetic photon will generate electronpositron pairs, for example, at least if there is a charged object such as a nucleus in the neighbourhood. A more luxuriant illustration of how the vacuum may be a source of matter is Hawking's account of the production of pairs of particles in the strong gravitational field of a gravitationally collapsed object, a black hole.

The novelty of the Higgs mechanism is that its raison d'être is so abstract. To demonstrate the reality of its existence would not merely provide the missing capstone for the electroweak theory, but also a more tangible starting point than there now is for the manipulation of the theory.

\section{Putting four forces together}

UNIFICATION has been in the air since the 1930s, when Einstein and Heisenberg separately advertised ambitions of this kind. Einstein, encouraged by the success of his theory of general relativity, was looking for similar ways of describing the quantum fields of particle physics. Heisenberg's goal, at the outset more limited, was to use similar language to describe nucleons (protons and neutrons) and fermions (electrons and their congeners).

A much more ambitious goal is now in the air: the unification of the four fundamental interactions in which particles can be engaged. Part of the enthusiasm stems from the success of the electroweak theory of Glashow, Salam and Weinberg, which uses a single framework to describe the behaviour of two interactions - the electromagnetic and the weak nuclear forces.

There has indeed been further progress in that direction, notably with the gauge theory of quantum chromodynamics, which is the theory of the strong nuclear interaction that binds quarks together in hadrons. (The strong interaction acts exclusively between quarks, and thus differs from the weak interaction, which can 'see' quarks as well as leptons.) So why not put them both together, for a start?

That is not just an idle ambition, but consonant with the expectation that, at high energy, a common description will be possible. After all, the 'strong' and the 'weak' nuclear interactions stand out from the electromagnetic force because of their short range. But the range of the weak force, for example, is a simple consequence of the large mass $(\sim 80 \mathrm{GeV})$ of the $\mathrm{W}^{ \pm}$bosons. In interactions in which the energy exchanged is much greater, the huge difference of mass between the bos- 